\title{
Proximal Point Algorithms for Finding a Zero of a Finite Sum of Monotone Mappings in Banach Spaces
}

\author{
H. Zegeye ${ }^{1}$ and N. Shahzad ${ }^{2}$ \\ ${ }^{1}$ Departement of Mathematics, University of Botswana, Private Bag 00704, Gaborone, Botswana \\ ${ }^{2}$ Department of Mathematics, King Abdulaziz University, P.O. Box 80203, Jeddah 21589, Saudi Arabia
}

Correspondence should be addressed to N. Shahzad; nshahzad@kau.edu.sa

Received 15 February 2013; Revised 30 March 2013; Accepted 31 March 2013

Academic Editor: Yisheng Song

Copyright (c) $2013 \mathrm{H}$. Zegeye and N. Shahzad. This is an open access article distributed under the Creative Commons Attribution License, which permits unrestricted use, distribution, and reproduction in any medium, provided the original work is properly cited.

We introduce an iterative process which converges strongly to a zero of a finite sum of monotone mappings under certain conditions. Applications to a convex minimization problem are included. Our theorems improve and unify most of the results that have been proved in this direction for this important class of nonlinear mappings.

\section{Introduction}

Let $C$ be a nonempty subset of a real Banach space $E$ with dual $E^{*}$. A mapping $A: C \rightarrow E^{*}$ is said to be monotone if for each $x, y \in C$, the following inequality holds:

$$
\langle x-y, A x-A y\rangle \geq 0 .
$$

A monotone mapping $A \subset E \times E^{*}$ is said to be maximal monotone if its graph is not properly contained in the graph of any other monotone mapping. We know that if $A$ is maximal monotone mapping, then $A^{-1}(0)$ is closed and convex (see [1] for more details).

Monotone mappings were introduced by Zarantonello [2], Minty [3], and Kačurovskiu [4]. The notion of monotone in the context of variational methods for nonlinear operator equations was also used by Vaŭnberg and Kačurovskiǐ [5]. The central problem is to iteratively find a zero of a finite sum of monotone mappings $A_{1}, A_{2}, \ldots, A_{N}$ in a Banach space $E$, namely, a solution to the inclusion problem

$$
0 \in\left(A_{1}+A_{2}+\cdots+A_{N}\right) x
$$

It is known that many physically significant problems can be formulated as problems of the type (2). For instance, a stationary solution to the initial value problem of the evolution equation

$$
\frac{\partial x}{\partial t}+F x \ni 0, \quad x(0)=x_{0}
$$

can be formulated as (2) when the governing maximal monotone $F$ is of the form $F:=A_{1}+A_{2}+\cdots+A_{N}$ (see, e.g., [6]). In addition, optimization problems often need [7] to solve a minimization problem of the form

$$
\min _{x \in E}\left\{f_{1}(x)+f_{2}(x)+\cdots+f_{N}(x)\right\},
$$

where $f_{i}, i=1,2, \ldots, N$ are proper lower semicontinuous convex functions from $E$ to the extended real line $\bar{R}:=$ $(-\infty, \infty]$. If in (2), we assume that $A_{i}:=\partial f_{i}$, for $i=$ $1,2, \ldots, N$, where $\partial f_{i}$ is the subdifferential operator of $f_{i}$ in the sense of convex analysis, then (4) is equivalent to (2). Consequently, considerable research efforts have been devoted to methods of finding approximate solutions (when they exist) of equations of the form (2) for a sum of a finite number of monotone mappings (see, e.g., [6, 8-12]).

A well-known method for solving the equation $0 \in A x$ in a Hilbert space $H$ is the proximal point algorithm: $x_{1}=x \in H$ and

$$
x_{n+1}=J_{r_{n}} x_{n}, \quad(n=1,2, \ldots),
$$


where $r_{n} \subset(0, \infty)$ and $J_{r}=(I+r A)^{-1}$ for all $r>0$. This algorithm was first introduced by Martinet [10]. In 1976, Rockafellar [11] proved that if $\liminf _{n \rightarrow \infty} r_{n}>0$ and $A^{-1}(0) \neq \emptyset$, then the sequence $\left\{x_{n}\right\}$ defined by (5) converges weakly to an element of $A^{-1}(0)$. Later, many researchers have studied the convergence of the sequence defined by (5) in Hilbert spaces; see, for instance, $[8,12-18]$ and the references therein.

In 2000, Kamimura and Takahashi [9] proved that for a maximal monotone mapping $A$ in a Hilbert spaces $H$ and $J_{r}=$ $(I+r A)^{-1}$ for all $r>0$, the sequence $\left\{x_{n}\right\}$ defined by

$$
x_{n+1}=\alpha_{n} x+\left(1-\alpha_{n}\right) J_{r_{n}} x_{n}, \quad n \geq 0,
$$

where $\left\{\alpha_{n}\right\} \subset[0,1]$ and $\left\{r_{n}\right\} \subset(0, \infty)$ satisfy certain conditions, called Halpern type, converges strongly to a point in $A^{-1}(0)$.

In a reflexive Banach space $E$ and for a maximal monotone mapping $A: E \rightarrow 2^{E^{*}}$, Reich and Sabach [19] proved that the sequence $\left\{x_{n}\right\}$ defined by

$$
\begin{gathered}
0=\xi_{n}+\lambda_{n}^{-1}\left(\nabla f\left(y_{n}\right)-\nabla f\left(x_{n}\right)\right), \quad \xi_{n} \in A y_{n}, \\
H_{n}=\left\{z \in E:\left\langle\xi_{n}, z-y_{n}\right\rangle \leq 0\right\}, \\
W_{n}=\left\{z \in E:\left\langle\nabla f\left(x_{0}\right)-\nabla f\left(x_{n}\right), z-x_{n}\right\rangle \leq 0\right\}, \\
x_{n+1}=\operatorname{Proj}_{H_{n} \cap W_{n}}^{f}\left(x_{0}\right), \quad n=1,2, \ldots,
\end{gathered}
$$

where $\lambda_{n}>0$ and $\operatorname{proj}_{C}^{f}$ is the Bergman projection of $E$ on to a closed and convex subset $C \subset E$ induced by a well-chosen convex function $f$, converges strongly to a point in $A^{-1}(0)$.

Furthermore, many authors (see, e.g., [12, 20-25]) have studied strong convergence of an iterative process of Halpern type or proximal type to a common zero of a finite family of maximal monotone mappings in Hilbert spaces (or in Banach spaces).

Regarding iterative solution of a zero of sum of two maximal monotone mappings, Lions and Mercier [6] introduced the nonlinear Douglas-Rachford splitting iterative algorithm which generates a sequence $\left\{v_{n}\right\}$ by the recursion

$$
v_{n+1}=J_{\lambda}^{A}\left(2 J_{\lambda}^{B}-I\right) v_{n}+\left(I-J_{\lambda}^{B}\right) v_{n},
$$

where $J_{\lambda}^{T}$ denotes the resolvent of a monotone mapping $T$; that is, $J_{\lambda}^{T}:=(I+\lambda T)^{-1}$. They proved that the nonlinear Douglas-Rachford algorithm (8) converges weakly to a point $v$, a solution of the inclusion,

$$
0 \in(A+B) x
$$

for $A+B$ maximal monotone mappings in Hilbert spaces.

A natural question arises whether we can obtain an iterative scheme which converges strongly to a zero of sum of a finite number of monotone mappings in Banach spaces or not?

Motivated and inspired by the work mentioned above, it is our purpose in this paper to introduce an iterative scheme (see (21)) which converges strongly to a zero of a finite sum of monotone mappings under certain conditions. Applications to a convex minimization problem are included. Our theorems improve the results of Lions and Mercier [6] and most of the results that have been proved in this direction.

\section{Preliminaries}

Let $E$ be a Banach space and let $S(E)=\{x \in E:\|x\|=1\}$. Then, a Banach space $E$ is said to be smooth provided that the limit

$$
\lim _{t \rightarrow 0} \frac{\|x+t y\|-\|x\|}{t}
$$

exists for each $x, y \in S(E)$. The norm of $E$ is said to be uniformly smooth if the limit (10) is attained uniformly for $(x, y)$ in $S(E) \times S(E)$ (see [1]).

The modulus of convexity of $E$ is the function $\delta_{E}:(0,2] \rightarrow$ $[0,1]$ defined by

$$
\delta_{E}(\epsilon):=\inf \left\{1-\left\|\frac{x+y}{2}\right\|:\|x\|=\|y\|=1 ; \epsilon=\|x-y\|\right\} .
$$

$E$ is called uniformly convex if and only if $\delta_{E}(\epsilon)>0$, for every $\epsilon \in(0,2]$ (see [26]).

Lemma 1 (see [27]). Let $E$ be a smooth, strictly convex, and reflexive Banach space. Let $C$ be a nonempty closed convex subset of $E$, and let $A: C \subset E \rightarrow E^{*}$ be a monotone mapping. Then, $A$ is maximal if and only if $R(J+r A)=E^{*}$, for all $r>0$, where $J$ is the normalized duality mapping from $E$ into $2^{E^{*}}$ defined, for each $x \in E$, by

$$
J x:=\left\{f^{*} \in E^{*}:\left\langle x, f^{*}\right\rangle=\|x\|^{2}=\left\|f^{*}\right\|^{2}\right\},
$$

where $\langle\cdot, \cdot\rangle$ denotes the generalized duality pairing between members of $E$ and $E^{*}$. We recall that $E$ is smooth if and only if $J$ is single valued (see [1]). If $E=H$, a Hilbert space, then the duality mapping becomes the identity map on $\mathrm{H}$.

Lemma 2 (see [27]). Let $E$ be a reflexive with $E^{*}$ as its dual. Let $A: D(A) \subseteq E \rightarrow E^{*}$, and let $B: D(B) \subseteq E \rightarrow$ $E^{*}$ be maximal monotone mappings. Suppose that $D(A) \cap$ int $D(B) \neq \emptyset$. Then, $A+B$ is a maximal monotone mapping.

Lemma 3 (see [28]). Let $E$ be a reflexive with $E^{*}$ as its dual. Let $A: D(A) \subseteq E \rightarrow E^{*}$ be maximal monotone mapping, and let $B: D(B) \subseteq E \rightarrow E^{*}$ be monotone mappings such that $D(B)=E, B$ is hemicontinuous (i.e., continuous from the segments in $E$ to the weak star topology in $\left.E^{*}\right)$ and carries bounded sets into bounded sets. Then, $A+B$ is maximal monotone mapping.

Let $E$ be a smooth Banach space with dual $E^{*}$. Let the Lyapunov function $\phi: E \times E \rightarrow \mathbb{R}$, introduced by Alber [29], be defined by

$$
\phi(y, x)=\|y\|^{2}-2\langle y, J x\rangle+\|x\|^{2}, \quad \text { for } x, y \in E,
$$


where $J$ is the normalized duality mapping from $E$ into $2^{E^{*}}$. If $E=H$, a Hilbert space, then (13) reduces to $\phi(x, y)=$ $\|x-y\|^{2}$, for $x, y \in H$.

Let $E$ be a reflexive, strictly convex, and smooth Banach space, and let $C$ be a nonempty closed and convex subset of $E$. The generalized projection mapping, introduced by Alber [29], is a mapping $\Pi_{C}: E \rightarrow C$ that assigns an arbitrary point $x \in E$ to the minimizer, $\bar{x}$, of $\phi(\cdot, x)$ over $C$; that is, $\Pi_{C} x=\bar{x}$, where $\bar{x}$ is the solution to the minimization problem

$$
\phi(\bar{x}, x)=\min \{\phi(y, x), y \in C\} .
$$

We know the following lemmas.

Lemma 4 (see [23]). Let $E$ be a real smooth and uniformly convex Banach space, and let $\left\{x_{n}\right\}$ and $\left\{y_{n}\right\}$ be two sequences of $E$. If either $\left\{x_{n}\right\}$ or $\left\{y_{n}\right\}$ is bounded and $\phi\left(x_{n}, y_{n}\right) \rightarrow 0$, as $n \rightarrow \infty$, then $x_{n}-y_{n} \rightarrow 0$, as $n \rightarrow \infty$.

Lemma 5 (see [29]). Let $C$ be a convex subset of a real smooth Banach space $E$, and let $x \in E$. Then $x_{0}=\Pi_{C} x$ if and only if

$$
\left\langle z-x_{0}, J x-J x_{0}\right\rangle \leq 0, \quad \forall z \in C .
$$

We make use of the function $V: E \times E^{*} \rightarrow \mathbb{R}$ defined by

$$
V\left(x, x^{*}\right)=\|x\|^{2}-2\left\langle x, x^{*}\right\rangle+\|x\|^{2}, \quad \forall x \in E, x^{*} \in E,
$$

studied by Alber [29]. That is, $V(x, y)=\phi\left(x, J^{-1} x^{*}\right)$, for all $x \in E$ and $x^{*} \in E^{*}$.

In the sequel, we will make use of the following lemmas.

Lemma 6 (see [29]). Let $E$ be a reflexive strictly convex and smooth Banach space with $E^{*}$ as its dual. Then,

$$
V\left(x, x^{*}\right)+2\left\langle J^{-1} x^{*}-x, y^{*}\right\rangle \leq V\left(x, x^{*}+y^{*}\right),
$$

for all $x \in E$ and $x^{*}, y^{*} \in E^{*}$.

Lemma 7 (see [30]). Let $E$ be a smooth and strictly convex Banach space, $C$ be a nonempty closed convex subset of $E$, and $A \subset E \times E^{*}$ be a maximal monotone mapping. Let $Q_{r}$ be the resolvent of $A$ defined by $Q_{r}=(J+r A)^{-1} J$, for $r>0$ and $\left\{r_{n}\right\}$ a sequence of $(0, \infty)$ such that $\lim _{n \rightarrow \infty} r_{n}=\infty$. If $\left\{x_{n}\right\}$ is a bounded sequence of $C$ such that $Q_{r_{n}} x_{n} \rightarrow z$, then $z \in A^{-1}(0)$.

Lemma 8 (see [31]). Let $E$ be a smooth and strictly convex Banach space, $C$ be a nonempty closed convex subset of $E$, and $A \subset E \times E^{*}$ be a maximal monotone mapping, and $A^{-1}(0)$ is nonempty. Let $Q_{r}$ be the resolvent of $A$ defined by $Q_{r}=$ $(J+r A)^{-1} J$, for $r>0$. Then, for each $r>0$

$$
\phi\left(p, Q_{r} x\right)+\phi\left(Q_{r} x, x\right) \leq \phi(p, x)
$$

for all $p \in A^{-1}(0)$ and $x \in C$.

Lemma 9 (see [32]). Let $\left\{a_{n}\right\}$ be a sequence of nonnegative real numbers satisfying the following relation:

$$
a_{n+1} \leq\left(1-\alpha_{n}\right) a_{n}+\alpha_{n} \delta_{n}, \quad n \geq n_{0},
$$

where $\left\{\alpha_{n}\right\} \subset(0,1)$ and $\left\{\delta_{n}\right\} \subset R$ satisfying the following conditions: $\lim _{n \rightarrow \infty} \alpha_{n}=0, \sum_{n=1}^{\infty} \alpha_{n}=\infty$, and $\lim \sup _{n \rightarrow \infty} \delta_{n} \leq 0$. Then, $\lim _{n \rightarrow \infty} a_{n}=0$.

Lemma 10 (see [33]). Let $\left\{a_{n}\right\}$ be the sequences of real numbers such that there exists a subsequence $\left\{n_{i}\right\}$ of $\{n\}$ such that $a_{n_{i}}<$ $a_{n_{i}+1}$, for all $i \in N$. Then, there exists a nondecreasing sequence $\left\{m_{k}\right\} \subset N$ such that $m_{k} \rightarrow \infty$, and the following properties are satisfied by all (sufficiently large) numbers $k \in N$ :

$$
a_{m_{k}} \leq a_{m_{k}+1}, \quad a_{k} \leq a_{m_{k}+1} .
$$

In fact, $m_{k}=\max \left\{j \leq k: a_{j}<a_{j+1}\right\}$.

\section{Main Result}

Theorem 11. Let $C$ and $D$ be nonempty, closed and convex subsets of a smooth and uniformly convex real Banach space $E$ with $E^{*}$ as its dual. Assume that $C \cap \operatorname{int}(D) \neq \emptyset$. Let $A_{1}: C \rightarrow$ $E^{*}$ and $A_{2}, A_{3}, \ldots, A_{N}: D \rightarrow E^{*}$ be maximal monotone mappings. Assume that $F:=\left(A_{1}+A_{2}+\cdots+A_{N}\right)^{-1}(0)$ is nonempty. Let $\left\{x_{n}\right\}$ be a sequence generated by

$$
x_{0}=w \in C, \text { chosen arbitrarily, }
$$

$$
x_{n+1}=J^{-1}\left(\alpha_{n} J w+\left(1-\alpha_{n}\right) J\left(J+r_{n} A\right)^{-1} J x_{n}\right), \quad \forall n \geq 0,
$$

where $A=A_{1}+A_{2}+\cdots+A_{N}, \alpha_{n} \in(0,1)$ and $\left\{r_{n}\right\}$ a sequence of $(0, \infty)$ satisfying: $\lim _{n \rightarrow \infty} \alpha_{n}=0, \sum_{n=1}^{\infty} \alpha_{n}=\infty$, and $\lim _{n \rightarrow \infty} r_{n}=\infty$. Then, $\left\{x_{n}\right\}$ converges strongly to $p=\Pi_{F}(w)$.

Proof. Observe that by Lemma 2, we have that $A_{2}+A_{3}+\cdots+$ $A_{N}$ is maximal monotone. In addition, since $C \cap \operatorname{int}(D) \neq \emptyset$, the same lemma implies that $A=A_{1}+A_{2}+\cdots+A_{N}$ is maximal monotone. Now, let $p=\Pi_{F}(w)$, and let $w_{n}:=$ $Q_{r_{n}} x_{n}:=\left(J+r_{n} A\right)^{-1} J x_{n}$. Then, we have that $x_{n+1}=J^{-1}\left(\alpha_{n} J w+\right.$ $\left.\left(1-\alpha_{n}\right) J w_{n}\right)$, and since $p \in A^{-1}(0)$, from Lemma 8 , we get that

$$
\phi\left(p, w_{n}\right)=\phi\left(p, Q_{r_{n}} x_{n}\right) \leq \phi\left(p, x_{n}\right) .
$$

Now from (21), property of $\phi$, and (22) we get that

$$
\begin{aligned}
\phi\left(p, x_{n+1}\right)= & \phi\left(p, J^{-1}\left(\alpha_{n} J w+\left(1-\alpha_{n}\right) J w_{n}\right)\right) \\
= & \|p\|^{2}-2\left\langle p, \alpha_{n} J w+\left(1-\alpha_{n}\right) J w_{n}\right\rangle \\
& +\left\|\alpha_{n} J w+\left(1-\alpha_{n}\right) J w_{n}\right\|^{2} \\
\leq & \|p\|^{2}-2 \alpha_{n}\langle p, J w\rangle-2\left(1-\alpha_{n}\right)\left\langle p, J w_{n}\right\rangle \\
& +\alpha_{n}\|J w\|^{2}+\left(1-\alpha_{n}\right)\left\|J w_{n}\right\|^{2} \\
= & \alpha_{n} \phi(p, w)+\left(1-\alpha_{n}\right) \phi\left(p, w_{n}\right) \\
\leq & \alpha_{n} \phi(p, w)+\left(1-\alpha_{n}\right) \phi\left(p, x_{n}\right) .
\end{aligned}
$$

Thus, by induction,

$$
\phi\left(p, x_{n+1}\right) \leq \max \left\{\phi(p, w), \phi\left(p, x_{0}\right)\right\}, \quad \forall n \geq 0,
$$


which implies that $\left\{x_{n}\right\}$ is bounded. In addition, using Lemma 6 and property of $\phi$, we obtain that

$$
\begin{aligned}
\phi\left(p, x_{n+1}\right)= & V\left(p, J x_{n+1}\right) \\
\leq & V\left(p, J x_{n+1}-\alpha_{n}(J w-J p)\right) \\
& -2\left\langle x_{n+1}-p,-\alpha_{n}(J w-J p)\right\rangle \\
= & \phi\left(p, J^{-1}\left(\alpha_{n} J p+\left(1-\alpha_{n}\right) J w_{n}\right)\right) \\
& +2 \alpha_{n}\left\langle x_{n+1}-p, J w-J p\right\rangle \\
\leq & \alpha_{n} \phi(p, p)+\left(1-\alpha_{n}\right) \phi\left(p, w_{n}\right) \\
& +2 \alpha_{n}\left\langle x_{n+1}-p, J w-J p\right\rangle \\
= & \left(1-\alpha_{n}\right) \phi\left(p, w_{n}\right)+2 \alpha_{n}\left\langle x_{n+1}-p, J w-J p\right\rangle \\
\leq & \left(1-\alpha_{n}\right) \phi\left(p, x_{n}\right)+2 \alpha_{n}\left\langle x_{n+1}-p, J w-J p\right\rangle .
\end{aligned}
$$

Furthermore, using property of $\phi$ and the fact that $\alpha_{n} \rightarrow 0$, as $n \rightarrow \infty$, imply that

$$
\begin{aligned}
\phi\left(w_{n}, x_{n+1}\right) & =\phi\left(w_{n}, J^{-1}\left(\alpha_{n} J w+\left(1-\alpha_{n}\right) J w_{n}\right)\right) \\
& \leq \alpha_{n} \phi\left(w_{n}, w\right)+\left(1-\alpha_{n}\right) \phi\left(w_{n}, w_{n}\right) \\
& \leq \alpha_{n} \phi\left(w_{n}, w\right)+\left(1-\alpha_{n}\right) \phi\left(w_{n}, w_{n}\right) \longrightarrow 0, \\
& \text { as } n \longrightarrow \infty,
\end{aligned}
$$

which implies from Lemma 4 that

$$
w_{n}-x_{n+1} \longrightarrow 0, \quad \text { as } n \longrightarrow \infty \text {. }
$$

Now, following the method of proof of Lemma 3.2 of Maing'e [33], we consider two cases.

Case 1. Suppose that there exists $n_{0} \in \mathbb{N}$ such that $\left\{\phi\left(p, x_{n}\right)\right\}$ is nonincreasing for all $n \geq n_{0}$. In this situation, $\left\{\phi\left(p, x_{n}\right)\right\}$ is convergent. Since $\left\{x_{n+1}\right\}$ is bounded and $E$ is reflexive, we choose a subsequence $\left\{x_{n_{i}+1}\right\}$ of $\left\{x_{n+1}\right\}$ such that $x_{n_{i}+1} \rightarrow z$ and $\lim \sup _{n \rightarrow \infty}\left\langle x_{n+1}-p, J w-J p\right\rangle=\lim _{i \rightarrow \infty}\left\langle x_{n_{i}+1}-p, J w-\right.$ $J p\rangle$. Then, from (27), we get that

$$
w_{n_{i}} \rightarrow z, \quad \text { as } i \longrightarrow \infty \text {. }
$$

Thus, by Lemma 7, we get that $z \in A^{-1}(0)$, and hence $z \in$ $F=\left(A_{1}+A_{2}+\cdots+A_{N}\right)^{-1}(0)$. Therefore, by Lemma 5 , we immediately obtain that $\lim \sup _{n \rightarrow \infty}\left\langle x_{n+1}-p, J w-J p\right\rangle=$ $\lim _{i \rightarrow \infty}\left\langle x_{n_{i}+1}-p, J w-J p\right\rangle=\langle z-p, J w-J p\rangle \leq 0$. It follows from Lemma 9 and (25) that $\phi\left(p, x_{n}\right) \rightarrow 0$, as $n \rightarrow \infty$. Consequently, $x_{n} \rightarrow p$.

Case 2. Suppose that there exists a subsequence $\left\{n_{i}\right\}$ of $\{n\}$ such that

$$
\phi\left(p, x_{n_{i}}\right)<\phi\left(p, x_{n_{i}+1}\right)
$$

for all $i \in \mathbb{N}$. Then, by Lemma 10, there exist a nondecreasing sequence $\left\{m_{k}\right\} \subset \mathbb{N}$ such that $m_{k} \rightarrow \infty$, satisfying

$$
\begin{array}{r}
\phi\left(p, x_{m_{k}}\right) \leq \phi\left(p, x_{m_{k}+1}\right), \\
\phi\left(p, x_{k}\right) \leq \phi\left(p, x_{m_{k}+1}\right), \\
\forall k \in \mathbb{N} .
\end{array}
$$

Thus, following the method of proof of Case 1, we obtain that

$$
\limsup _{k \rightarrow \infty}\left\langle x_{m_{k}+1}-p, J w-J p\right\rangle \leq 0 .
$$

Then, from (25), we have that

$$
\begin{aligned}
\phi\left(p, x_{m_{k}+1}\right) \leq & \left(1-\alpha_{m_{k}}\right) \phi\left(p, x_{m_{k}}\right) \\
& +2 \alpha_{m_{k}}\left\langle x_{m_{k}+1}-p, J w-J p\right\rangle .
\end{aligned}
$$

Now, inequalities (30) and (32) imply that

$$
\begin{aligned}
\alpha_{m_{k}} \phi\left(p, x_{m_{k}}\right) \leq & \phi\left(p, x_{m_{k}}\right)-\phi\left(p, x_{m_{k}+1}\right) \\
& +2 \alpha_{m_{k}}\left\langle x_{m_{k}+1}-p, J w-J p\right\rangle \\
\leq & 2 \alpha_{m_{k}}\left\langle x_{m_{k}+1}-p, J w-J p\right\rangle .
\end{aligned}
$$

In particular, since $\alpha_{m_{k}}>0$, we get

$$
\phi\left(p, x_{m_{k}}\right) \leq 2\left\langle x_{m_{k}+1}-p, J w-J p\right\rangle .
$$

Then, from (31), we obtain $\phi\left(p, x_{m_{k}}\right) \rightarrow 0$, as $k \rightarrow \infty$. This together with (32) gives $\phi\left(p, x_{m_{k}+1}\right) \rightarrow 0$, as $k \rightarrow \infty$. But $\phi\left(p, x_{k}\right) \leq \phi\left(p, x_{m_{k}+1}\right)$, for all $k \in N$; thus, we obtain that $x_{k} \rightarrow p$. Therefore, from the above two cases, we can conclude that $\left\{x_{n}\right\}$ converges strongly to $p$, and the proof is complete.

Theorem 12. Let $C$ be a nonempty, closed, and convex subset of a smooth and uniformly convex real Banach space $E$ with $E^{*}$ as its dual. Let $A_{1}: C \rightarrow E^{*}$ be maximal monotone mapping, and let $A_{2}, A_{3}, \ldots, A_{N}: E \rightarrow E^{*}$ be bounded and hemicontinuous monotone mappings. Assume that $F:=\left(A_{1}+\right.$ $\left.A_{2}+\cdots+A_{N}\right)^{-1}(0)$ is nonempty. Let $\left\{x_{n}\right\}$ be a sequence generated by

$$
x_{0}=w \in C, \text { chosen arbitrarily, }
$$

$$
x_{n+1}=J^{-1}\left(\alpha_{n} J w+\left(1-\alpha_{n}\right) J\left(J+r_{n} A\right)^{-1} J x_{n}\right), \quad \forall n \geq 0,
$$

where $A=A_{1}+A_{2}+\cdots+A_{N}, \alpha_{n} \in(0,1)$ and $\left\{r_{n}\right\}$ is a sequence of $(0, \infty)$ satisfying: $\lim _{n \rightarrow \infty} \alpha_{n}=0, \sum_{n=1}^{\infty} \alpha_{n}=\infty$, and $\lim _{n \rightarrow \infty} r_{n}=\infty$. Then, $\left\{x_{n}\right\}$ converges strongly to $p=$ $\Pi_{F}(w)$.

Proof. By Lemma 3, we have that $A=A_{1}+A_{2}+\cdots+A_{N}$ is maximal monotone, and hence following the method of proof of Theorem 11, we obtain the required assertion. 
If in Theorem 12, we assume that $A_{i}$, for $i=2, \ldots, N$, are continuous monotone mappings, then $A_{i}^{\prime} s$ are hemicontinuous, and hence we get the following corollary.

Corollary 13. Let $C$ be a nonempty, closed, and convex subset of a smooth and uniformly convex real Banach space $E$ with $E^{*}$ as its dual. Let $A_{1}: C \rightarrow E^{*}$ be a maximal monotone mapping, and let $A_{2}, A_{3}, \ldots, A_{N}: E \rightarrow E^{*}$ be bounded and continuous monotone mappings. Assume that $F:=\left(A_{1}+A_{2}+\right.$ $\left.\cdots+A_{N}\right)^{-1}(0)$ is nonempty. Let $\left\{x_{n}\right\}$ be a sequence generated by

$$
\begin{gathered}
x_{0}=w \in C, \text { chosen arbitrarily, } \\
x_{n+1}=J^{-1}\left(\alpha_{n} J w+\left(1-\alpha_{n}\right) J\left(J+r_{n} A\right)^{-1} J x_{n}\right), \quad \forall n \geq 0,
\end{gathered}
$$

where $A=A_{1}+A_{2}+\cdots+A_{N}, \alpha_{n} \in(0,1)$ and $\left\{r_{n}\right\}$ a sequence of $(0, \infty)$ satisfying: $\lim _{n \rightarrow \infty} \alpha_{n}=0, \sum_{n=1}^{\infty} \alpha_{n}=\infty$, and $\lim _{n \rightarrow \infty} r_{n}=\infty$. Then, $\left\{x_{n}\right\}$ converges strongly to $p=\Pi_{F}(w)$.

If in Theorem 12, we assume that $A_{i}$, for $i=2, \ldots, N$, are uniformly continuous monotone mapping, then $A_{i}^{\prime} s$ are bounded and hemicontinuous, and hence we get the following corollary.

Corollary 14. Let $C$ be a nonempty, closed, and convex subset of a smooth and uniformly convex real Banach space $E$ with $E^{*}$ as its dual. Let $A_{1}: C \rightarrow E^{*}$ be a maximal monotone mapping, and let $A_{2}, A_{3}, \ldots, A_{N}: E \rightarrow E^{*}$ be monotone uniformly continuous mappings. Assume that $F:=\left(A_{1}+A_{2}+\right.$ $\left.\cdots+A_{N}\right)^{-1}(0)$ is nonempty. Let $\left\{x_{n}\right\}$ be a sequence generated by

$$
\begin{gathered}
x_{0}=w \in C, \text { chosen arbitrarily, } \\
x_{n+1}=J^{-1}\left(\alpha_{n} J w+\left(1-\alpha_{n}\right) J\left(J+r_{n} A\right)^{-1} J x_{n}\right), \quad \forall n \geq 0,
\end{gathered}
$$

where $A=A_{1}+A_{2}+\cdots+A_{N}, \alpha_{n} \in(0,1)$ and $\left\{r_{n}\right\}$ a sequence of $(0, \infty)$ satisfying: $\lim _{n \rightarrow \infty} \alpha_{n}=0, \sum_{n=1}^{\infty} \alpha_{n}=\infty$, and $\lim _{n \rightarrow \infty} r_{n}=\infty$. Then, $\left\{x_{n}\right\}$ converges strongly to $p=\Pi_{F}(w)$.

If in Theorem 12 we assume that $A_{i} \equiv 0$, for $i=2, \ldots, N$, then we get the following corollary.

Corollary 15. Let $C$ be a nonempty, closed, and convex subset of a smooth and uniformly convex real Banach space E. Let A : $C \rightarrow E^{*}$ be a maximal monotone mapping. Assume that $F:=$ $A^{-1}(0)$ is nonempty. Let $\left\{x_{n}\right\}$ be a sequence generated by

$$
\begin{gathered}
x_{0}=w \in C, \text { chosen arbitrarily, } \\
x_{n+1}=J^{-1}\left(\alpha_{n} J w+\left(1-\alpha_{n}\right) J\left(J+r_{n} A\right)^{-1} J x_{n}\right), \quad \forall n \geq 0,
\end{gathered}
$$

where $\alpha_{n} \in(0,1)$ and $\left\{r_{n}\right\}$ a sequence of $(0, \infty)$ satisfying: $\lim _{n \rightarrow \infty} \alpha_{n}=0, \sum_{n=1}^{\infty} \alpha_{n}=\infty$, and $\lim _{n \rightarrow \infty} r_{n}=\infty$. Then, $\left\{x_{n}\right\}$ converges strongly to $p=\Pi_{F}(w)$.
If $E=H$, a real Hilbert space, then $E$ is smooth and uniformly convex real Banach space. In this case, $J=I$, identity map on $H$ and $\Pi_{C}=P_{C}$, projection mapping from $H$ onto $C$. Thus, the following corollaries follow from Theorems 11 and 12.

Corollary 16. Let $C$ and $D$ be nonempty, closed, and convex subsets of a real Hilbert space $H$. Assume that $C \cap \operatorname{int}(D) \neq \emptyset$. Let $A_{1}: C \rightarrow H$, and let $A_{2}, A_{3}, \ldots, A_{N}: D \rightarrow H$ be maximal monotone mappings. Assume that $F:=\left(A_{1}+A_{2}+\right.$ $\left.\cdots+A_{N}\right)^{-1}(0)$ is nonempty. Let $\left\{x_{n}\right\}$ be a sequence generated by

$$
\begin{gathered}
x_{0}=w \in C, \text { chosen arbitrarily, } \\
x_{n+1}=\alpha_{n} w+\left(1-\alpha_{n}\right)\left(I+r_{n} A\right)^{-1} x_{n}, \quad \forall n \geq 0,
\end{gathered}
$$

where $A=A_{1}+A_{2}+\cdots+A_{N}, \alpha_{n} \in(0,1)$ and $\left\{r_{n}\right\}$ a sequence of $(0, \infty)$ satisfying: $\lim _{n \rightarrow \infty} \alpha_{n}=0, \sum_{n=1}^{\infty} \alpha_{n}=\infty$, and $\lim _{n \rightarrow \infty} r_{n}=\infty$. Then, $\left\{x_{n}\right\}$ converges strongly to $p=P_{F}(w)$.

Corollary 17. Let $C$ be a nonempty, closed, and convex subset of a real Hilbert space $H$. Let $A_{1}: C \rightarrow H$ be a maximal monotone mapping, and let $A_{2}, A_{3}, \ldots, A_{N}: H \rightarrow H$ be bounded, hemicontinuous, and monotone mappings. Assume that $F:=\left(A_{1}+A_{2}+\cdots+A_{N}\right)^{-1}(0)$ is nonempty. Let $\left\{x_{n}\right\}$ be a sequence generated by

$$
\begin{gathered}
x_{0}=w \in C, \text { chosen arbitrarily, } \\
x_{n+1}=\alpha_{n} w+\left(1-\alpha_{n}\right)\left(I+r_{n} A\right)^{-1} x_{n}, \quad \forall n \geq 0,
\end{gathered}
$$

where $A=A_{1}+A_{2}+\cdots+A_{N}, \alpha_{n} \in(0,1)$ and $\left\{r_{n}\right\}$ a sequence of $(0, \infty)$ satisfying: $\lim _{n \rightarrow \infty} \alpha_{n}=0, \sum_{n=1}^{\infty} \alpha_{n}=\infty$, and $\lim _{n \rightarrow \infty} r_{n}=\infty$. Then, $\left\{x_{n}\right\}$ converges strongly to $p=P_{F}(w)$.

Corollary 18. Let $C$ be a nonempty, closed, and convex subset of a real Hilbert space $H$. Let $A_{1}: C \rightarrow H$ be a maximal monotone mapping, and let $A_{2}, A_{3}, \ldots, A_{N}: H \rightarrow H$ be uniformly continuous monotone mappings. Assume that $F:=$ $\left(A_{1}+A_{2}+\cdots+A_{N}\right)^{-1}(0)$ is nonempty. Let $\left\{x_{n}\right\}$ be a sequence generated by

$$
\begin{gathered}
x_{0}=w \in C, \text { chosen arbitrarily, } \\
x_{n+1}=\alpha_{n} w+\left(1-\alpha_{n}\right)\left(I+r_{n} A\right)^{-1} x_{n}, \quad \forall n \geq 0,
\end{gathered}
$$

where $A=A_{1}+A_{2}+\cdots+A_{N}, \alpha_{n} \in(0,1)$ and $\left\{r_{n}\right\}$ a sequence of $(0, \infty)$ satisfying: $\lim _{n \rightarrow \infty} \alpha_{n}=0, \sum_{n=1}^{\infty} \alpha_{n}=\infty$, and $\lim _{n \rightarrow \infty} r_{n}=\infty$. Then, $\left\{x_{n}\right\}$ converges strongly to $p=P_{F}(w)$.

\section{Application}

In this section, we study the problem of finding a minimizer of a continuously Fréchet differentiable convex functional in Banach spaces. The followings are deduced from Theorems 11 and 12 .

Theorem 19. Let $C$ and $D$ be a nonempty, closed, and convex subsets of a smooth and uniformly convex real Banach space E. 
Let $C$ int $(D) \neq \emptyset$. Let $f$ be a continuously Fréchet differentiable convex functional, and let $\nabla f$ be maximal monotone on $C$. Let $g$ be a continuously Fréchet differentiable convex functional, and let $\nabla g$ be maximal monotone on $D$. Assume that $F:=(\nabla f+$ $\nabla g)^{-1}(0)=\left\{z \in E: f(z)+g(z)=\inf _{y \in E}\{f(y)+g(y)\}\right\} \neq \emptyset$. Let $\left\{x_{n}\right\}$ be a sequence generated by

$$
\begin{gathered}
x_{0} \in C \text { chosen arbitrarily, } \\
x_{n+1}=J^{-1}\left(\alpha_{n} J w+\left(1-\alpha_{n}\right) J\left(J+r_{n}(\nabla f+\nabla g)\right)^{-1} J x_{n}\right),
\end{gathered}
$$

where $\alpha_{n} \in(0,1)$ and $\left\{r_{n}\right\}$ a sequence of $(0, \infty)$ satisfying: $\lim _{n \rightarrow \infty} \alpha_{n}=0, \sum_{n=1}^{\infty} \alpha_{n}=\infty$, and $\lim _{n \rightarrow \infty} r_{n}=\infty$. Then, $\left\{x_{n}\right\}$ converges strongly to an element of $F$.

Theorem 20. Let $C$ be a nonempty, closed, and convex subset of a smooth and uniformly convex real Banach space. Let $f$ be a continuously Fréchet differentiable convex functional, and let $\nabla f$ be maximal monotone on $C$. Let $g$ be a continuously Fréchet differentiable convex functional, and let $\nabla g$ be bounded, hemicontinuous, and monotone on $E$ with $F:=(\nabla f+$ $\nabla g)^{-1}(0)=\left\{z \in E: f(z)+g(z)=\inf _{y \in E}\{f(y)+g(y)\}\right\} \neq \emptyset$. Let $\left\{x_{n}\right\}$ be a sequence generated by

$$
\begin{gathered}
x_{0} \in C \text { chosen arbitrarily, } \\
x_{n+1}=J^{-1}\left(\alpha_{n} J w+\left(1-\alpha_{n}\right) J\left(J+r_{n}(\nabla f+\nabla g)\right)^{-1} J x_{n}\right),
\end{gathered}
$$

where $\alpha_{n} \in(0,1)$ and $\left\{r_{n}\right\}$ a sequence of $(0, \infty)$ satisfying: $\lim _{n \rightarrow \infty} \alpha_{n}=0, \sum_{n=1}^{\infty} \alpha_{n}=\infty$, and $\lim _{n \rightarrow \infty} r_{n}=\infty$. Then, $\left\{x_{n}\right\}$ converges strongly to an element of $F$.

Remark 21. Our results provide strong convergence theorems for finding a zero of a finite sum of monotone mappings in Banach spaces and hence extend the results of Rockafellar [11], Kamimura and Takahashi [9], and Lions and Mercier [6].

\section{Acknowledgments}

The authors thank the referee for his comments that considerably improved the paper. The research of N. Shahzad was partially supported by Deanship of Scientific Research (DSR), King Abdulaziz University, Jeddah, Saudi Arabia.

\section{References}

[1] W. Takahashi, Nonlinear Functional Analysis, Kindikagaku, Tokyo, Japan, 1988.

[2] E. H. Zarantonello, "Solving functional equations by contractive averaging," Tech. Rep. 160, Mathematics Research Centre, Univesity of Wisconsin, Madison, Wis, USA, 1960.

[3] G. J. Minty, "Monotone (nonlinear) operators in Hilbert space," Duke Mathematical Journal, vol. 29, pp. 341-346, 1962.

[4] R. I. Kačurovskiŭ, "On monotone operators and convex functionals," Uspekhi Mathematicheskikh Nauk, vol. 15, no. 4, pp. 213-215, 1960.

[5] M. M. Vaĭnberg and R. I. Kačurovskiŭ, "On the variational theory of non-linear operators and equations," Doklady Akademii Nauk SSSR, vol. 129, pp. 1199-1202, 1959.
[6] P.-L. Lions and B. Mercier, "Splitting algorithms for the sum of two nonlinear operators," SIAM Journal on Numerical Analysis, vol. 16, no. 6, pp. 964-979, 1979.

[7] P. L. Combettes and V. R. Wajs, "Signal recovery by proximal forward-backward splitting," Multiscale Modeling \& Simulation, vol. 4, no. 4, pp. 1168-1200, 2005.

[8] H. Brézis and P.-L. Lions, "Produits infinis de résolvantes," Israel Journal of Mathematics, vol. 29, no. 4, pp. 329-345, 1978.

[9] S. Kamimura and W. Takahashi, "Approximating solutions of maximal monotone operators in Hilbert spaces," Journal of Approximation Theory, vol. 106, no. 2, pp. 226-240, 2000.

[10] B. Martinet, "Régularisation d'inéquations variationnelles par approximations successives," Revue Française d'Informatique et de Recherche Opérationnelle, vol. 4, pp. 154-158, 1970.

[11] R. T. Rockafellar, "Monotone operators and the proximal point algorithm," SIAM Journal on Control and Optimization, vol. 14, no. 5, pp. 877-898, 1976.

[12] M. V. Solodov and B. F. Svaiter, "Forcing strong convergence of proximal point iterations in a Hilbert space," Mathematical Programming, vol. 87, no. 1, pp. 189-202, 2000.

[13] H. H. Bauschke, E. Matoušková, and S. Reich, "Projection and proximal point methods: convergence results and counterexamples," Nonlinear Analysis: Theory, Methods \&Applications, vol. 56, no. 5, pp. 715-738, 2004.

[14] R. E. Bruck and S. Reich, "Nonexpansive projections and resolvents of accretive operators in Banach spaces," Houston Journal of Mathematics, vol. 3, no. 4, pp. 459-470, 1977.

[15] O. Güler, "On the convergence of the proximal point algorithm for convex minimization," SIAM Journal on Control and Optimization, vol. 29, no. 2, pp. 403-419, 1991.

[16] P.-L. Lions, "Une méthode itérative de résolution d'une inéquation variationnelle," Israel Journal of Mathematics, vol. 31, no. 2, pp. 204-208, 1978.

[17] O. Nevanlinna and S. Reich, "Strong convergence of contraction semigroups and of iterative methods for accretive operators in Banach spaces," Israel Journal of Mathematics, vol. 32, no. 1, pp. 44-58, 1979.

[18] G. B. Passty, "Ergodic convergence to a zero of the sum of monotone operators in Hilbert space," Journal of Mathematical Analysis and Applications, vol. 72, no. 2, pp. 383-390, 1979.

[19] S. Reich and S. Sabach, "A strong convergence theorem for a proximal-type algorithm in reflexive Banach spaces," Journal of Nonlinear and Convex Analysis, vol. 10, no. 3, pp. 471-485, 2009.

[20] Y. Censor and S. Reich, "Iterations of paracontractions and firmly nonexpansive operators with applications to feasibility and optimization," Optimization, vol. 37, no. 4, pp. 323-339, 1996.

[21] L. Hu and L. Liu, "A new iterative algorithm for common solutions of a finite family of accretive operators," Nonlinear Analysis: Theory, Methods \& Applications, vol. 70, no. 6, pp. 2344-2351, 2009.

[22] S. Reich and S. Sabach, "Two strong convergence theorems for a proximal method in reflexive Banach spaces," Numerical Functional Analysis and Optimization, vol. 31, no. 1-3, pp. 2244, 2010.

[23] S. Kamimura and W. Takahashi, "Strong convergence of a proximal-type algorithm in a Banach space," SIAM Journal on Optimization, vol. 13, no. 3, pp. 938-945, 2002.

[24] H. Zegeye and N. Shahzad, "Strong convergence theorems for a common zero for a finite family of $m$-accretive mappings," Nonlinear Analysis: Theory, Methods \& Applications, vol. 66, no. 5, pp. 1161-1169, 2007. 
[25] H. Zegeye and N. Shahzad, "Approximating common solution of variational inequality problems for two monotone mappings in Banach spaces," Optimization Letters, vol. 5, no. 4, pp. 691704, 2011.

[26] K. Goebel and S. Reich, Uniform Convexity, Hyperbolic Geometry, and Nonexpansive Mappings, vol. 83 of Monographs and Textbooks in Pure and Applied Mathematics, Marcel Dekker, New York, NY, USA, 1984.

[27] R. T. Rockafellar, "On the maximality of sums of nonlinear monotone operators," Transactions of the American Mathematical Society, vol. 149, pp. 75-88, 1970.

[28] F. E. Browder, "Nonlinear maximal monotone operators in Banach space," Mathematische Annalen, vol. 175, pp. 89-113, 1968.

[29] Y. I. Alber, "Metric and generalized projection operators in Banach spaces: properties and applications," in Theory and Applications of Nonlinear Operators of Accretive and Monotone Type, A. G. Kartsatos, Ed., vol. 178 of Lecture Notes in Pure and Applied Mathematics, pp. 15-50, Dekker, New York, NY, USA, 1996.

[30] K. Aoyama, F. Kohsaka, and W. Takahashi, "Proximal point methods for monotone operators in Banach spaces," Taiwanese Journal of Mathematics, vol. 15, no. 1, pp. 259-281, 2011.

[31] S. Kamimura, F. Kohsaka, and W. Takahashi, "Weak and strong convergence theorems for maximal monotone operators in a Banach space," Set-Valued Analysis, vol. 12, no. 4, pp. 417-429, 2004.

[32] H.-K. Xu, "Another control condition in an iterative method for nonexpansive mappings," Bulletin of the Australian Mathematical Society, vol. 65, no. 1, pp. 109-113, 2002.

[33] P.-E. Maingé, "Strong convergence of projected subgradient methods for nonsmooth and nonstrictly convex minimization," Set-Valued Analysis, vol. 16, no. 7-8, pp. 899-912, 2008. 


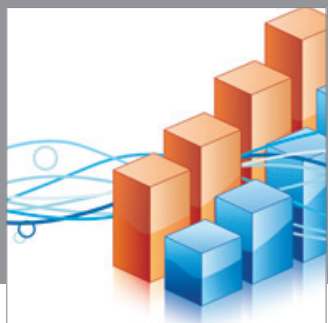

Advances in

Operations Research

mansans

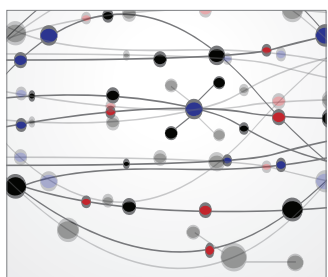

The Scientific World Journal
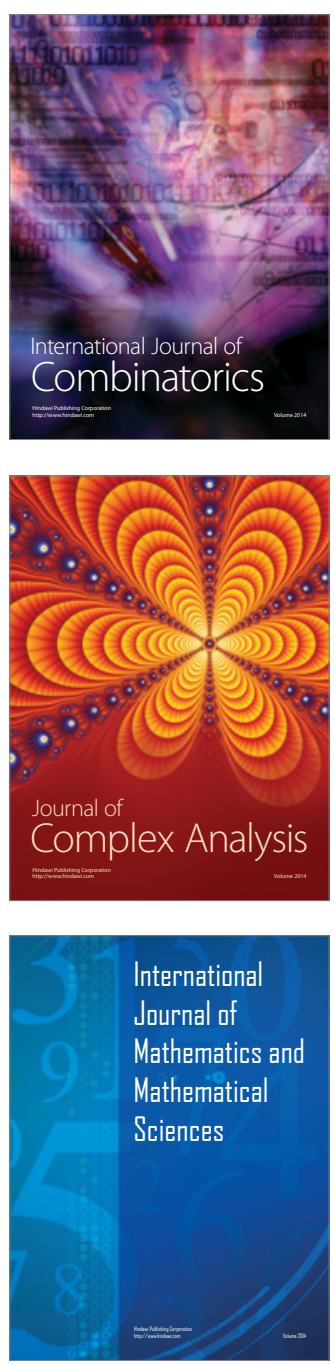
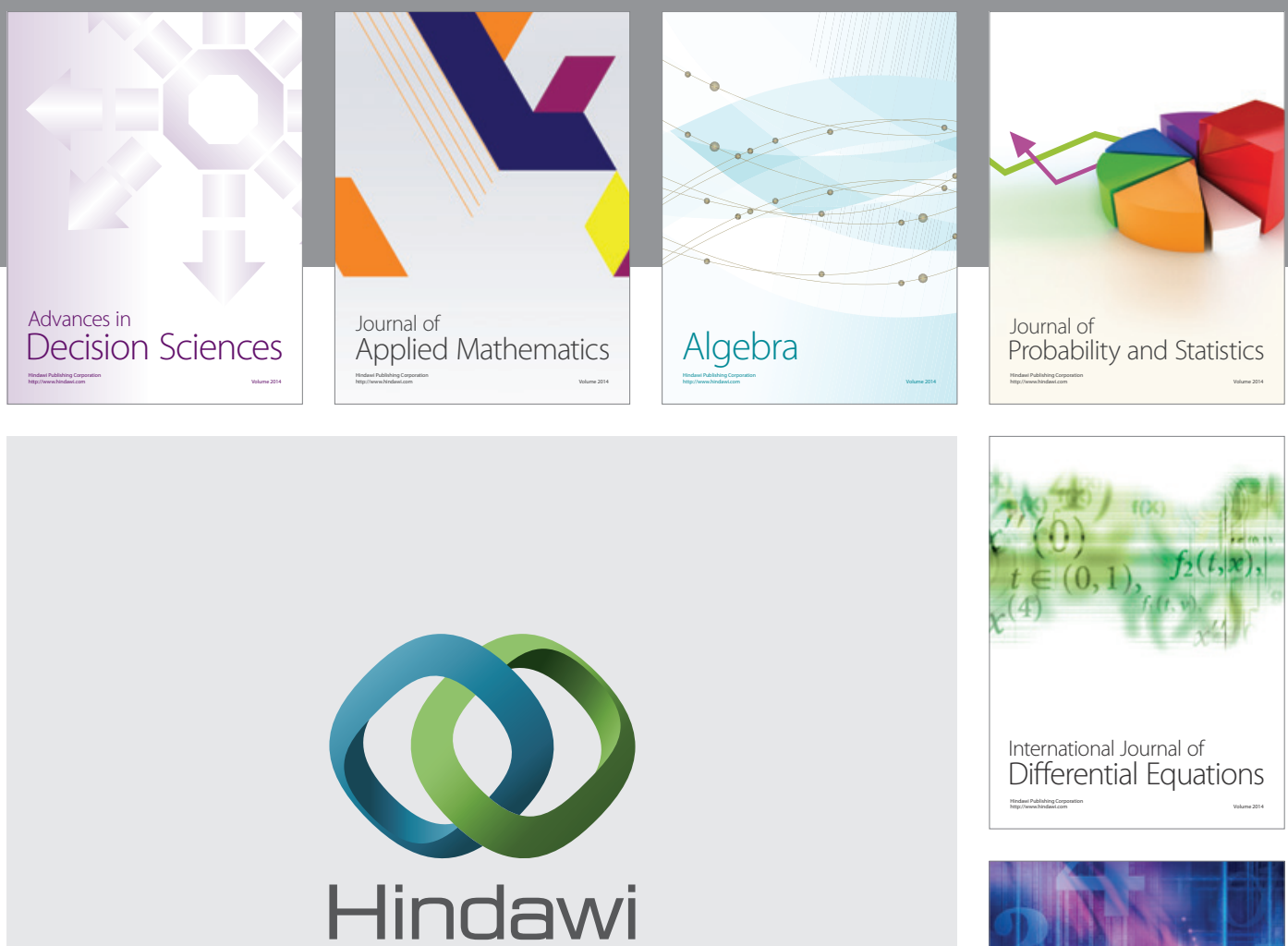

Submit your manuscripts at http://www.hindawi.com
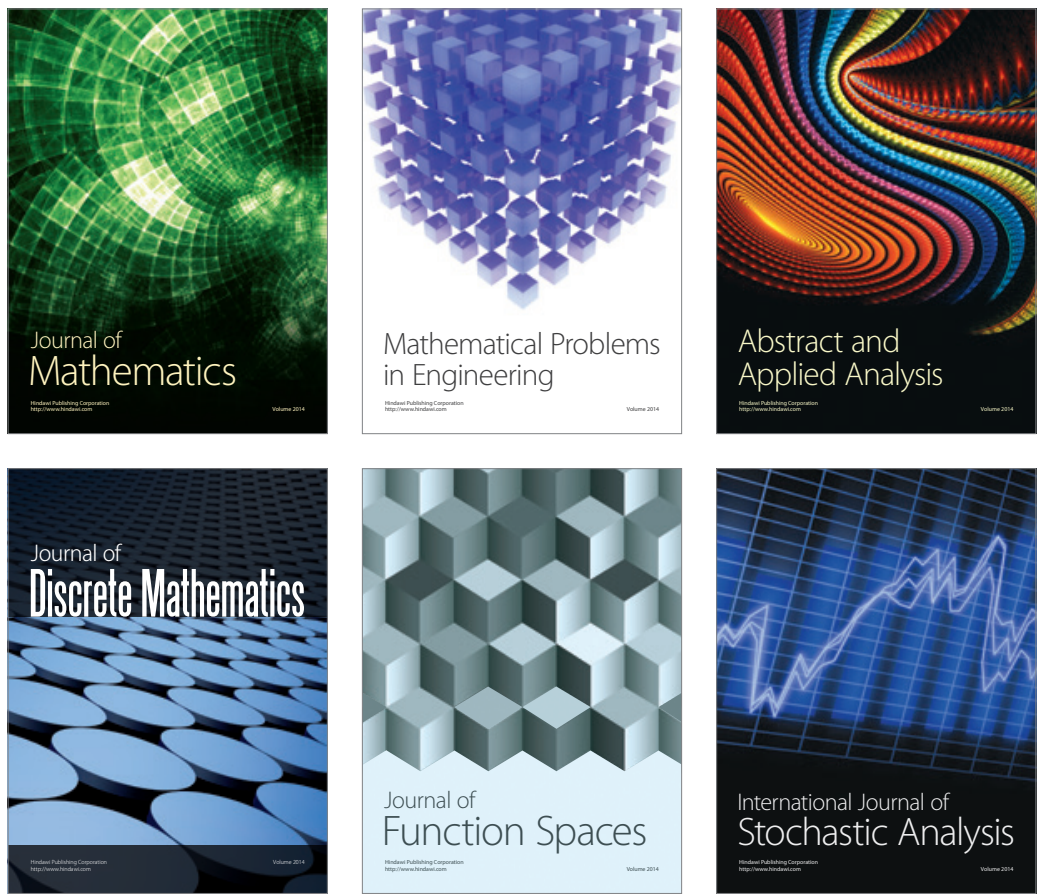

Journal of

Function Spaces

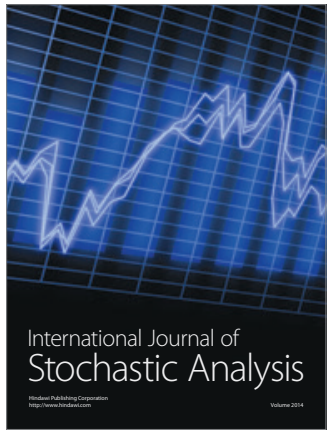

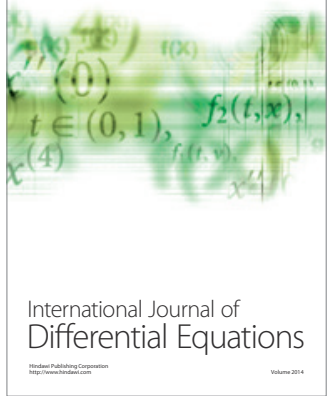
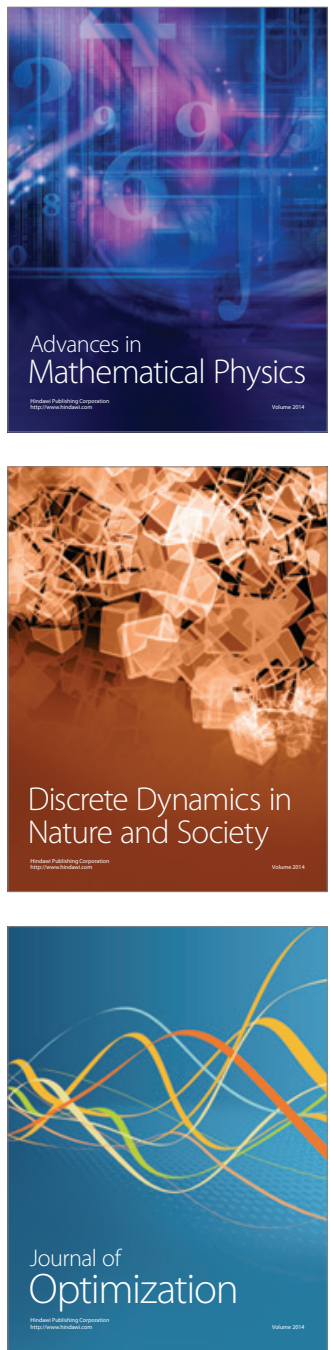